\title{
Compact Coupler Designs for Quantum Optical Circuits Produced by Direct UV Writing
}

\author{
D.O. Kundys ${ }^{1}$, J.C. Gates ${ }^{1}$, C.B.E. Gawith ${ }^{1}$, B.J. Smith ${ }^{2}$, J.S. Lundeen ${ }^{2}$, I.A. Walmsley ${ }^{2}$, P.G.R. Smith ${ }^{1}$ \\ 1. Optoelectronics Research Centre, University of Southampton, Highfield, Southampton, SO17 1BJ, UK \\ 2. University of Oxford, Clarendon Lab, Oxford, OX1 3PU, UK \\ dmk@orc.soton.ac.uk
}

Integrated planar lightwave circuits (PLCs) provide a promising route to small-scale quantum optical networks [1]. Recent work on quantum logic gates using silica-based PLCs has highlighted the opportunities afforded by the ability to coherently manipulate degrees of freedom at the level of single photons [2]. Increasingly complex waveguide networks are required for linear optics quantum computing (LOQC), a route towards small scale quantum information processing [3]. This approach uses quantum interference between photons and measurements with feedforward to implement the nonlinear interactions between photons that are required for information processing. A major issue in developing such circuits is the internal loss and the coupling efficiency of input photon from optical fibers.

Direct UV writing (DUVW) provides a technique for creating silica-based Planar Lightwave Circuits (PLCs) offering the advantages of fibre-compatibility, low propagation loss, rapid prototyping flexibility, and the ability to locally modulate refractive index with a precision of few nanometres [4]. It is a particularly flexible technique for fabricating silica-based planar waveguide structures because it uniquely allows control of refractive index along the propagation length of a channel waveguide to create tailored optical devices such as efficient Xcouplers [5], which cannot be fabricated lithographically. Definition of our waveguide structures is achieved via a UV-induced refractive index change in a photosensitive planar layer, and the relative translation of a focused UV beam and silica-on-silicon substrate by computer-controlled motion provides an accurate and reproducible method of fabricating integrated optical elements [4].

At present PLCs for quantum optics application must be operated at wavelength of $\sim 800 \mathrm{~nm}$ (not the more common $1550 \mathrm{~nm}$ of telecoms waveguides) where the avalanche silicon photodiode single-photon counting units are at their maximum efficiency of $\sim 70 \%$. This provides a challenge to the technology in terms of fabrication precision and robustness. Here we demonstrate that it is possible. In this work, we report the fabrication of DUVW structures appropriate for quantum optical networks, and discuss the use of X-couplers for a more compact device layout with improved fibre compatibility, v-groove launch, and polarisation control. These devices are well suited to quantum interference applications.

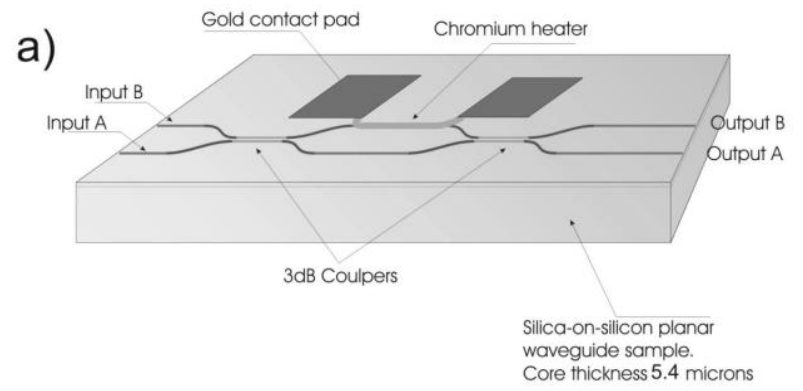

b)

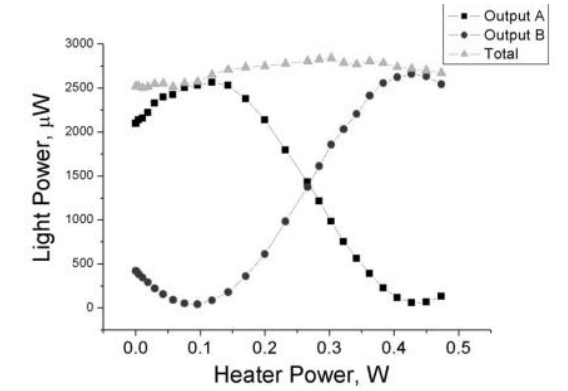

Fig.1a Schematic of a DUVW MZI with thermal tuning element.

Fig.1b Characterization of MZI output modulation via thermooptical phase control.

Figure 1a illustrates our simple device design incorporating the key elements of a quantum circuit. Figure 1b provides the optical characterisation results from a DUVW quantum circuit device as a ratio of measured power from the two output ports of the device. Transfer of the output power from one port to the other is shown as a function of electrically detuned thermo-optical modulation. Our devices demonstrate high-visibility classical interference, a symmetrical response from each of the coupling ports, and a fast thermo-optical tuning response.

The ability to combine optical elements into a quantum network using DUVW silica-on-silicon photonic chips offers a unique opportunity for successful implementation of PLCs in quantum information processing.

\section{References}

[1] I. A. Walmsley and M. G. Raymer, “Toward Quantum-Information Processing with Photons”, Science 307, 1733 (2005).

[2] A. Politi, M. J. Cryan, J. G. Rarity, S. Yu, J. L. O'Brien, "Silica-on-Silicon Waveguide Quantum Circuits" Science 320, 646 (2008).

[3] E. Knill, R. Laflamme G. J. Milburn, "A Scheme for Efficient Computation with Linear Optics" Nature 409, 46 (2001).

[4] G. D. Emmerson, S. P. Watt, C. B. E. Gawith, V. Albanis, M. Ibsen, R. B. Williams, P. G. R. Smith, "Fabrication of directly UV-written channel waveguides with simultaneously defined integral Bragg gratings," Elec. Lett. 38, 1531 (2002).

[5] D.O. Kundys, J.C. Gates, S. Dasgupta, N.G.R. Broderick, C.B.E. Gawith, P.G.R. Smith, "Use of Cross-Couplers to Decrease Size of UV Written Photonic Circuits" manuscript in preparation. 